\title{
Advance Physiotherapeutic Rehabilitation Approach for Hand Functions in a Giant Cell Tumour Patient- A Case Report on Palliative Physiotherapy
}

\author{
Pooja Ajay Shah ${ }^{1}$, Sneha Vilas Nemade², Waqar M. Naqvi ${ }^{3}$
}

\begin{abstract}
${ }^{1}$ Department of Community Health Sciences, Data Meghe Institute of Medical Sciences, Sawangi, Meghe, Wardha, Maharashtra, India. ${ }^{2}$ Department of Community Health Sciences, Data Meghe Institute of Medical Sciences, Sawangi, Meghe, Wardha, Maharashtra, India. ${ }^{3}$ Department of Community Health Sciences, Data Meghe Institute of Medical Sciences, Sawangi, Meghe, Wardha, Maharashtra, India.
\end{abstract}

\section{INTRODUCTION}

Giant cell tumour is a benign tumour of the bone. It is aggressive locally and has low metastatic potential.(1) Following lower end of femur and upper tibia, distal end of radius is the third most evident location for giant cell tumour (GCT) and about 10 percent of GCT involves distal radius.(2) GCT have often been identified as difficult to manage, mainly due to their close proximity to multiple tendons, median nerves, radial arteries and carpals.(3) Surgical treatment includes extended curettage of bone and replacing the cavity with bone graft, or excision of the tumour followed by reconstruction of bone with autograft or allograft.(1) Wrist fusion after ulnar translocation results in reduced range of pronation and supination movements in the forearm initially and mainly flexion and extension of the wrist joint, lag of the extensor pollicis brevis and longus tendons, abductor pollicis longus, stiffness and the risk of infections at the site.(6) Physiotherapy has been shown to be effective in the post-surgery treatment of patients. Nonetheless, the therapeutic program involves passive movement to active movements, manual joint mobilization, progressive resisted exercises, muscle energy technique and electrotherapy with adequate patient education. This has been shown to help improve patient outcomes and alleviate pain and recover full range of motion.(7)

The distal end of the radius is the third most apparent site for giant cell tumour (GCT) after the lower end of the femur and upper tibia and about 10 per cent of GCT includes distal radius. After ulnar translocation and wrist arthrodesis the full range of motion cannot be regained at the wrist joint and which makes the case unique, physical therapy has been shown to be helpful for improving patient performance and quality of life in post-surgical situations. Patient main concerns were pain and oedema around wrist with loss of strength, power and range of motion. Main clinical findings found in this case were severe reduction in of range of motion at CMC, MCP and IP joints. Reduced strength in hand muscles. Loss of superficial sensation over C6, C7 in affected extremity (right). Giant Cell Tumour that was confirmed by X-Ray and histopathology as well as MRI. Nerve injury confirmed by Nerve Conduction Velocity. Therapeutic interventions are found to be beneficial in these type cases. The patient was able to achieve 4/6 activities of daily living and gain functional range of motion after continuous 10 weeks of intensive systematic physiotherapy treatment program.
Corresponding Author:

Waqar M. Naqvi,

Professor and HOD,

Department of Community Health

Physiotherapy,

Ravi Nair Physiotherapy College, Wardha, Maharashtra, India.

E-mail: waqar.naqvi@dmimsu.edu.in

DOI: $10.14260 /$ jemds/2020/368

Financial or Other Competing Interests: None.

How to Cite This Article:

Shah PA, Nemade SV, Naqvi WM. Advance physiotherapeutic rehabilitation approach for hand functions in a giant cell tumour patient- a case report on palliative physiotherapy. J. Evolution Med. Dent. Sci. 2020;9(21):1675-1678,

10.14260/jemds/2020/368

Submission 22-03-2020,

Peer Review 05-05-2020,

Acceptance 12-05-2020,

Published 25-05-2020.

\section{(i)}




\section{PRESENTATION OF CASE}

A 65-year-old male retired government employee with righthand dominance visited the orthopaedics department who was referred by physiotherapy department with complaints of pain and swelling at the right wrist and trouble performing upper extremity activities. Patient gives history of slip and fall 3 years ago and was diagnosed with a fracture around the right wrist. Two years later the patient started experiencing pain over the right wrist which gradually increased over time. He visited a hospital where X-ray, CT-scan and Doppler were performed and he was diagnosed with Giant Cell Tumour.
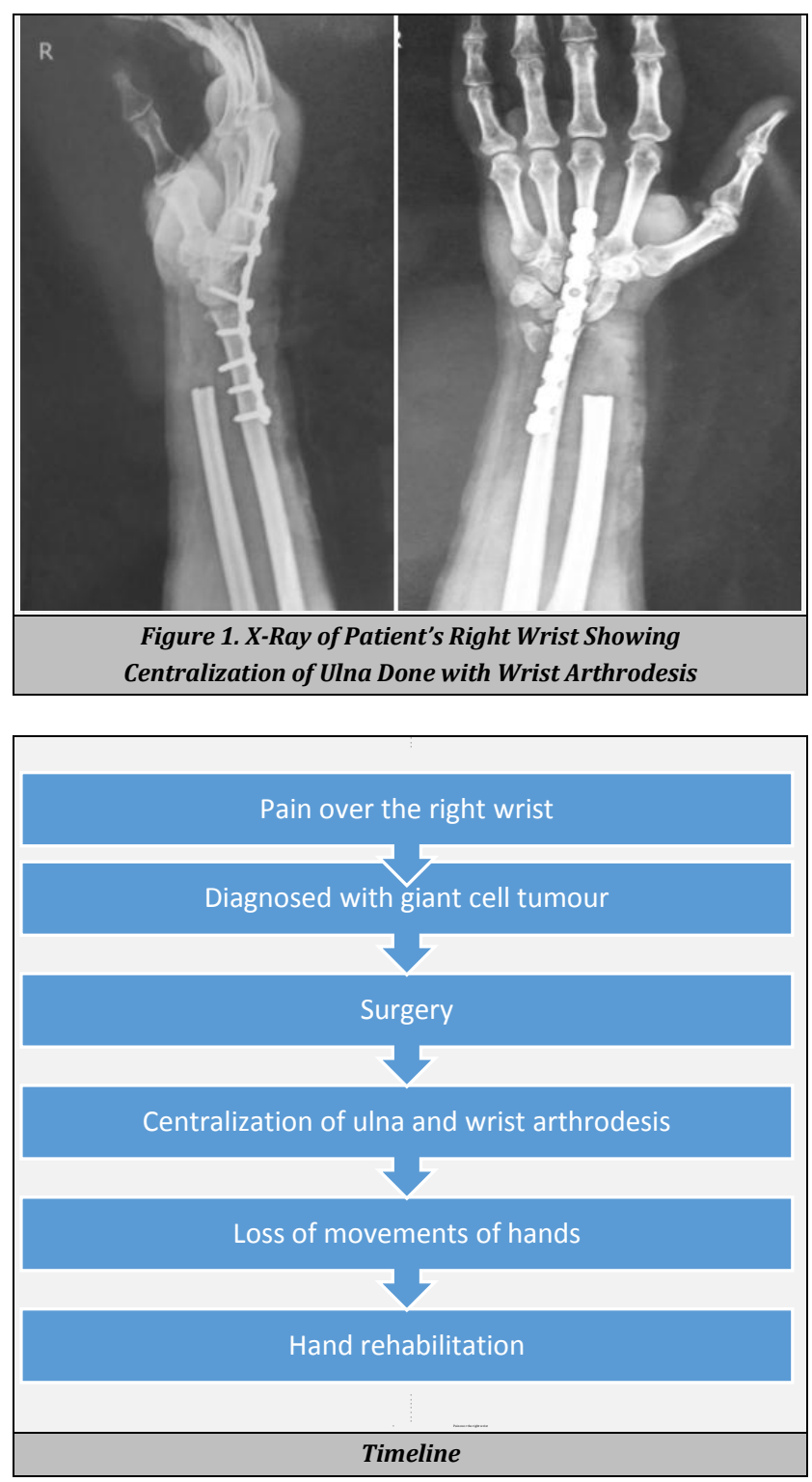

\section{Clinical Findings}

Patient was examine in sitting position with both shoulder at same level. On inspection, patient keeps right shoulder adducted, elbow flexed approx. 30 , forearm mid-pronated, wrist in neutral and interphalangeal joint mid-flexed position.. Presence of swelling on palmer and dorsal aspect of hand. On palpation we did found that the local temperature was raised and the oedema was non pitting type.. Whereas on Neurological assessment, we found that there is loss of superficial sensations (light touch, pin prick and temperature) at C6, C7 in right upper extremity. Grade 1-impairment in right extremity. In Myotome assessment, it was limited due to the arthrodesis, however grossly impaired from C6-C8 in right extremity and the deep tendon reflexes were found intact.

\begin{tabular}{|ccc|}
\hline Shoulder & Flexor & $4+$ \\
& Abductors & $4+$ \\
Elbow & Flexor & $2+$ \\
& Extensor & NT \\
Wrist & Flexor & NT \\
& Extensor & NT \\
Thumb & Flexion & $1+$ \\
& Extension & 0 \\
Fingers & Flexion & $2+$ \\
& extension \\
\hline
\end{tabular}

\begin{tabular}{|cccc|}
\hline Joint & Active & Passive & Limitation \\
Elbow flexion & $30-120$ & $0-120$ & $2 / 10$ (pain) \\
Wrist flexion & NT & NT & Unable to perform \\
Extension & NT & NT & Unable to perform \\
Radial deviation & NT & NT & Unable to perform \\
Ulnar deviation & NT & NT & Unable to perform \\
Forearm supination & $0-20$ & $0-25$ & $5 / 10$ (pain) \\
Pronation & $0-50$ & $0-60$ & $-3 / 10$ (pain) \\
Thumb flexion & 0 & $0-50$ & (no motor control) \\
Extension & 0 & $0-15$ & (no motor control) \\
Abduction & 0 & $0-50$ & (no motor control) \\
Adduction & 0 & $0-20$ & (no motor control) \\
Opposition & 0 & Grade 9 & (no motor control) \\
MCP joint flexion & $0-20$ & $0-60$ & 2/10 (pain) \\
MCP joint extension & $0-5$ & $0-15$ & (reduced motor control) \\
\hline \multicolumn{4}{c}{ Range of Motion } \\
\hline
\end{tabular}

\section{Diagnostic Challenges}

Non Compliance ratio is higher when the patient is old and from rural area. Due to lack of access and ignorance to proper healthcare facilities patient visited late to physiotherapy department, that resulted in stiffness at wrist, thumb and MCP joints. Post-surgery patient was advised physiotherapy but he did not do so because of lack of patient education.

\section{DISCUSSION OF MANAGEMENT}

Patient was treated surgically where resection of tumour with centralization of ulna and wrist arthrodesis was done. Postoperatively patient was treated with antibiotics, antiplatelets, analgesics and LMWH. Physiotherapy consultation was advised to the patient, but he visited the physiotherapy department 4 weeks after removal of elbow slab.

Post-operatively patient had chief complaints of pain and swelling around right wrist which patient described as dullaching with a rating of 5/10 on NPRS at rest, also reduced joint range (ROM), difficulty during performing daily living activities such as holding objects gripping, combing, grooming, self-hygiene and unable to perform activities related to occupation.

Patient underwent physiotherapy session for duration of 10 weeks, 5 days a week. Sessions included patient education, thermotherapy, electrotherapeutic modalities, range of motion exercises, sensory re-education techniques, progressive resisted exercises, joint mobilisation and stretching.

Patient was advised physiotherapy after removal of slab but patient visited physiotherapy department late by 4 weeks. 


\section{Description \\ Week 1}

Application of paraffin wax bath for 20-30 min duration in a breaking of three sessions that helps to relax muscle and reduce pain and oedema. It was applied using brush technique. EMS (electrical muscle stimulator) galvanic stimulation for 30 counts initially. Range of motion exercises - Active shoulder ROM exercises, active and active assisted ROM exercises for elbow and passive ROM exercise for forearm including movements thumb and fingers. ROM exercises were given to prevent contractures and deformity. 10 repetitions 2 times a day was given. Stretching of thumb flexors and extensors along with finger flexors and extensors were done keeping forearm in mid prone position. Stretch force was hold for 15-30 seconds, 5 repetitions, 2 times a day.

\section{Week 2- 4}

Paraffin Wax therapy was continued but duration was reduced as swelling was significantly reduced. Range of motion exercises were continued only repetitions were increased to 20 times 2 times a day. Graded joint mobilisation was started at grade I and II (Maitland) dose at 3 bouts of 30 seconds interval, followed by stretching for 3-5 minutes. Sensory reeducation was done by utilizing different modalities (desensitisation and discriminative sensory re-education). Electrical muscle stimulation given with intermediate galvanic current for 40-60 repetitions and intensity of current was according to patient tolerance level. Finger spring exerciser with least resistance was given to the patient to improve and facilitate opposition.

\section{Week 5-8}

Thermotherapy discontinued as pain and swelling subsided. Range of motion exercise was continued. Graded mobilisation grade III and IV was combined with stretching holding for 1530 seconds and progressed according to patient response. Isometric strengthening exercises were initiated for shoulder, elbow with $10 \mathrm{sec}$ hold and 10 repetitions to increase the strength. Patient motivation was done by asking him to initiate flexion, extension, supination and pronation and resistance was given by therapist and holding it for 10 seconds. Electrical muscle contraction was changed to faradic stimulation for 30 60 repetitions and intensity according to patient tolerance level.

\section{Week 8-10}

Finger spring exerciser with high resistance level was given to improve and strengthen opponens muscle. Gel ball exerciser started to improve grip. Static stretching for elbow flexors and active range of motion were done. Static stretching for thumb and finger flexors and extensors continued to prevent contracture and stimulate Golgi tendon organ. Functional task oriented exercise were started for reducing difficulty with daily activities.

\section{Home Programme}

The patient were put on home exercise programme after completion of session of physiotherapy. Patient was asked to do rubber band exercises at home. Rubber band was tied over the thumb and index finger and the patient was asked to stretch the rubber band for improving the extension of index finger and thumb and also to improve the thumb abduction.

Rubber band tied to thumb and alternatively with each finger also improved the opposition. The patient was asked to use a sponge ball at home to increase grip strength. Selfstretching was taught to patient. The patient was asked for follow up once in 15 days.

\section{Follow Up and Outcomes}

- Table 1 shows initial and final range of motion evaluation.

- Table 2 shows initial and final strength evaluation.

- After 10 weeks of intervention a linear growth in improvement was reported.

- Remarkable decreased in level of pain on NPRS from 5/10 to $1 / 10$ during rest

- DASH score was remarkably improved from $99 \%$ to $33 \%$.

- PSFS - Patient capacity increased in activities like grooming, writing, eating, holding a bag.

\begin{tabular}{|ccccc|}
\hline & \multicolumn{2}{c}{ Pre } & \multicolumn{2}{c|}{ Post } \\
Joint & Active & Passive & Active & Passive \\
Elbow flexion & $30-120$ & $0-120$ & $0-120$ & $0-120$ \\
Wrist flexion & NT & NT & NT & NT \\
Extension & NT & NT & NT & NT \\
Radial deviation & NT & NT & NT & NT \\
Ulnar deviation & NT & NT & NT & NT \\
Forearm supination & $0-20$ & $0-25$ & $0-25$ & $0-50$ \\
Pronation & $0-50$ & $0-60$ & $0-60$ & $0-70$ \\
Thumb flexion & 0 & $0-50$ & $0-30$ & $0-55$ \\
Extension & 0 & $0-15$ & $0-8$ & $0-15$ \\
Abduction & 0 & $0-50$ & $0-20$ & $0-50$ \\
Adduction & 0 & $0-20$ & $0-10$ & $0-20$ \\
Opposition & 0 & Grade 9 & Grade 5 & Grade 9 \\
MCP joint flexion & $0-20$ & $0-60$ & $0-40$ & $0-65$ \\
MCP joint extension & $0-5$ & $0-15$ & $0-15$ & $0-20$ \\
\hline & Table 1. Range of Motion \\
\hline \multicolumn{4}{c}{} \\
\hline
\end{tabular}

\begin{tabular}{|cccc|}
\hline \multirow{3}{*}{ Shoulder } & Flexor & Pre & Post \\
& Abductors & $4+$ & $4+$ \\
Elbow & Flexor & $4+$ & $4+$ \\
& Extensor & $2+$ & $3+$ \\
Wrist & Flexor & NT & NT \\
& Extensor & NT & NT \\
Thumb & Flexion & $1+$ & NT \\
& Extension & 0 & $2+$ \\
Fingers & Flexion & $2+$ & $3+$ \\
& extension & $2+$ & $4+$ \\
\hline & Table 2. Strength & \\
\hline
\end{tabular}

\section{PSFS- Patient Specific Functional Scale}

- $\quad$ Pre score-1/10

- Post score - 5/10

\section{NPRS- Numeric Pain Rating Scale}

- $\quad$ Pre score-5/10

- Post score-1/10

\section{Limitations}

Delayed patient presentation led to stiffness and oedema around the wrist joint. Lack of access to health care services had raised the difficulty of the situation; if patient was treated with physiotherapy earlier, maximum range of motion would 
have been achieved. The care plan for each patient differs and this procedure listed is not common for all of these types of cases.

\section{DISCUSSION}

In this case, patient presented to physiotherapy department with complaints of pain, oedema, stiffness, reduced range of motion, reduced strength, loss of sensation over palmar and dorsal aspect of hand and difficulty in upper extremity movement. After clinical evaluation a plan of care was made which included thermotherapy through paraffin wax bath. The paraffin wax bath is basically effective in improving blood circulation and promoting relaxation of muscles ${ }^{(8)}$ thus it helps reducing stiffness and as well as oedema. Application of heat potentiates the effect of stretching of a number of muscle groups on improving ROM. Heating has had a beneficial effect on both the acute ROM gain and the persistent ROM gain that is noticeable after multiple treatments in healthy individuals. Electrical muscle stimulator was used initially with intermittent galvanic current to stimulate the motor point and muscle function. The patient had little hand function, thus the increase in the available range of motion and increased strength of extensors may have improved the potential for functional ability. ${ }^{(9)}$

ROM exercises were initially initiated to advance passively towards active assisted exercises, and then free exercises. There is evidence to support the importance of specific exercise regimens to minimize impairments and enhance the function of the upper limbs. Graded joint mobilization (Maitland) was given in grades I and II initially, 3 bouts at intervals of 30 seconds. It help reduce pain as well break adhesions and reduce stiffness. After mobilization, stretching was done for 8-10 minutes further mobilization was progressed to grade III and IV. The use of Thera-putty assisted the patient with improvement in sensation as well improvement of pincer grip. Use of different modalities like finger spring exerciser, gel hand exerciser and rubber band help the patient to improve strength of hand muscles, power grip and make the hand functionally able to the activities of daily living.

\section{CONCLUSIONS}

The patient was provided a comprehensive recovery plan that helped him by relieving pain, reducing oedema, increase strength, and range of motion, post-operatively. Patient was able to conduct everyday living tasks with routine exercises, and 4/6 targets were achieved. In conclusion, this case report demonstrates that patient with post-operative giant cell tumour with centralization of ulna doesn't experience full recovery after conservative management. However, functional range of motion can be achieved. Taking lessons from this case report is that early patient education is of utmost important. Lack of patient awareness and carelessness can increase complications. Doing something instead of nothing helps to restore some function in the patient.

\section{REFERENCES}

[1] Hussin P, Singh V. Giant cell tumour of distal radius: a case report and description of surgical technique. The Internet Journal of Orthopedic Surgery 2007;8(2).

[2] Wang Y, Min L, Lu M, et al. The functional outcomes and complications of different reconstruction methods for Giant cell tumour of the distal radius: comparison of osteoarticular allograft and three-dimensional-printed prosthesis. BMC Musculoskelet Disord 2020;21(1):69.

[3] Chalidis BE, Dimitriou CG. Modified ulnar translocation technique for the reconstruction of giant cell tumour of the distal radius. Orthopedics 2008;31(6):608.

[4] Puri A, Gulia A, Agarwal MG, et al. Ulnar translocation after excision of a Campanacci grade-3 giant-cell tumour of the distal radius: an effective method of reconstruction. J Bone Joint Surg Br 2010;92(6):875-9.

[5] Salunke AA, Shah J, Warikoo V, et al. Giant cell tumour of distal radius treated with ulnar translocation and wrist arthrodesis: What are the functional outcomes? J Orthop Surg (Hong Kong) 2017;25(1):2309499016684972.

[6] Meena DK, Thalanki S, Sharma SB. Wrist fusion through centralisation of the ulna for recurrent giant cell tumour of the distal radius. J Orthop Surg (Hong Kong) 2016;24(1):84-7.

[7] Hays PL, Rozental TD. Rehabilitative strategies following hand fractures. Hand Clinics 2013;29(4):585-600.

[8] Sibtain F, Khan A, Shakil-ur-Rehman S. Efficacy of paraffin wax bath with and without joint mobilization techniques in rehabilitation of post-traumatic stiff hand. PakJ Med Sci 2013;29(2):647-50.

[9] Baker LL, Yeh C, Wilson D, et al. Electrical stimulation of wrist and fingers for hemiplegic patients. Physical Therapy 1979;59(12):1495-9. 